\title{
An analysis of energy consumption Equilibrium in MIMO wireless sensor networks
}

\author{
Dong-Lai Hao, Dong-Hong Xie and Lin Zhou \\ Department of Information transmission, Xi' an Communication Institute, Xi'an,710106, \\ China \\ Email:hao_dl@126.com
}

\begin{abstract}
Based on analyzing the total energy consumption of cooperative transmission and the proportion of energy consumption of each node, this paper presents a method for calculating equilibrium degree of the node energy consumption and its balance scheme. Comparing with the traditional cooperative MISO scheme and the ideal feedback cooperative scheme, the results of analysis and simulation shows that the proposed scheme can effectively reduce the total energy consumption and improve energy balance degree. Energy consumption of nodes in the network is relatively balanced, and the network life time can be also extended.

Keywords: Wireless Sensor Networks; Multiple-Input Multiple-Output; Energy Consumption Balance.
\end{abstract}

\section{Introduction}

Maximizing the lifetime of network is the primary consideration factors in the design of wireless sensor network, especially, the cluster head node and collaborative nodes of power without effective allocation, and in selecting cooperative nodes, not considering the node residual energy is considered cluster nodes and cluster head node between the channel quality, and do not consider the cluster remote node and the number according to the fusion center between the channel condition. From the literature [1], we can know that the transmission power of the transmitter can be adjusted to improve the BER performance such as Alamouti codes under the total power constraint. To adjust the transmission power of the transmitter, the channel state information should be feed back to the transmitter. In the literature [2], it is pointed out that the performance of MIMO system can be improved by using full channel state information, but it is not suitable to be applied to wireless sensor networks with limited energy. In order to compromise the complexity and performance, D J Love in the literature [3] that the use of limited feedback can make the MIMO system to obtain a higher transmission performance. But it is difficult to get all the channel 
information, especially in the limited energy wireless sensor networks. The life of the network can be defined as the time of death for first node in the network[4], if energy consumption of the node move to the other nodes, the life of network will be extended. In other words, to make the network life longer, we must make the energy balance of the nodes in the network. Therefore, under the condition of the minimum total energy consumption[5], this paper makes a concrete analysis of the equilibrium degree of the node energy consumption.

\section{System Model}

We show that the energy consumption is expressed as follows:

$\eta=\left\|\xi_{\mathrm{CNint}, i}-\xi_{\mathrm{CNres}, i}-\mathrm{E}\left[\xi_{\mathrm{CNint}, i}-\xi_{\mathrm{CNres}, i}\right]\right\|_{2}^{-1}$

Where $\xi_{\mathrm{CNint}, i}$ is the initial energy for the node $i, \xi_{\mathrm{CNres}, i}$ is the remaining energy for the node $\mathrm{i}$ after the given data is sent, For the convenience of analysis, note that the $\mathrm{CH}$ as node $1, \mathrm{CCHs}$ is noted as $2,3, \cdots, N_{\mathrm{C}}+1,\|\|_{2}$ is the Euclidean norm. The larger $\eta$ is, the energy consumption of nodes in the cluster is more balanced, and the more unbalanced on the contrary.

\section{Energy Consumption Balance Algorithm}

To ensure the system performance, energy consumption for $\mathrm{CH}$ and $\mathrm{CNs}$ is smaller than $\mathrm{CCH}$ with poor channel quality when looking for $\mathrm{CCHs}$ once again. Thus achieving the effect of energy consumption balance and extending the life of the network. Because the energy consumption of each frame transmission is different according to the different channel quality, it is difficult to use the mathematical expression, so the algorithm can be described as follows:

Step1: The constellation size $b$ and the number of $\mathrm{CCHs} N_{\mathrm{C}}$ are determined according to the mutual information between $\mathrm{CH}$ and $\mathrm{CNs}$ when the cluster formed. Initializing transmission power coefficient of $\mathrm{CH}$ and $\mathrm{CCHs}$ $\beta_{i, 1}=1 /\left(N_{\mathrm{C}}+1\right), \quad i=1,2, \cdots, N_{\mathrm{C}}+1$, the initial energy of $\mathrm{CH}$ is $\xi_{\mathrm{CHres}}=\xi_{\mathrm{CHint}}$ the initial energy of CCHs is $\xi_{\mathrm{CCHres}, i}=\xi_{\mathrm{CCint}, i}$, and the initial energy of CCHs is $\xi_{\mathrm{CNres}, j}=\xi_{\mathrm{CNint}, j}, j=N_{\mathrm{C}}+1, \cdots, N$.

Step2: For $k=1: F_{\text {Num }} N_{\text {frame }}$,otherwise to step7. Calculate the transmission energy consumption of each node $\xi_{\text {trans-long }, i, k}$.If $b$ is even number, 
then $\xi_{\text {trans-long }, i, k}=\beta_{i, k}\left(\frac{\left(2\left(2^{b}-1\right) N_{0}\right)}{3 b}\right)\left(\frac{4\left(1-2^{-b / 2}\right)}{\left(b \delta_{\mathrm{b}}\right)} \prod_{i=1}^{N_{\mathrm{C}}+1} \bar{\beta}_{i}^{-1}\right)^{1 /\left(N_{\mathrm{C}}+1\right)} ;$ If $b$ is odd, and $b=1$, then $\xi_{\text {trans-long }, i, k}=\beta_{i, k} N_{0} \delta_{\mathrm{b}}^{-1 /\left(N_{\mathrm{C}}+1\right)}\left(\prod_{i=1}^{N_{\mathrm{C}}+1} \bar{\beta}_{i}^{-1}\right)^{-1 /\left(N_{\mathrm{C}}+1\right)}$, otherwise

$$
\xi_{\text {trans-long, }, i, k}=\beta_{i, k} \frac{2\left(2^{b}-1\right) N_{0}}{3 b}\left(\frac{4}{b \delta_{\mathrm{b}}} \prod_{i=1}^{N_{\mathrm{C}}+1} \bar{\beta}_{i}^{-1}\right)^{1 /\left(N_{\mathrm{C}}+1\right)} ;
$$

Step3: Calculate $E_{\mathrm{CHres}}=\xi_{\mathrm{CHres}}, E_{\mathrm{CCHres}, i}=\xi_{\mathrm{CCHres}, i}$, the residual energy of $\mathrm{CH}, \quad \xi_{\mathrm{CHres}}=E_{\mathrm{CHres}}-\left(\xi_{\text {loacl }}+\xi_{\text {trans-long }, i, k}+\xi_{\text {Tx-local }}+\xi_{\text {Rx-local }}\right)$ and the residual energy of the cooperative $\mathrm{CCHs}$ $\xi_{\mathrm{CCHres}, i}=E_{\mathrm{CCHres}, i}-\left(\xi_{\text {trans-long }, i, k}+\xi_{\mathrm{Tx} \text {-local }}+\xi_{\mathrm{Rx} \text {-local }}\right)$;

Step4: Update feedback coefficient $\beta_{i, k+1}=\left|h_{i}\right|^{2} /\|\mathbf{H}\|_{\mathrm{F}}^{2}$, if $\left|h_{i}\right|^{2}>g_{\text {thr }}$, then go to Step2, otherwise update $\mathrm{CCHs}$;

Step5: For $m=1: N$, calculating the residual energy of $\mathrm{CH}$ and $\mathrm{CNs}$ without cooperation $\xi_{\mathrm{CHres}}$ and $\xi_{\mathrm{CNres}, j}$, that is $\xi_{\text {CHres }}=\xi_{\text {CHres }}-l_{\mathrm{B}}\left(\xi_{\text {trans }}+\xi_{\text {elec }}\right)-l_{\text {mc }} \xi_{\text {elec }}$,and

$\xi_{\mathrm{CNres}, j}=\xi_{\mathrm{CNres}, j}-l_{\mathrm{B}} \xi_{\text {elec }}-l_{\mathrm{mc}}\left(\xi_{\text {trans }}+\xi_{\text {elec }}\right)$;

Step6: Go to step2;

Step7: According to

$\eta=\left\|\xi_{\mathrm{CNint}, i}-\xi_{\mathrm{CNres}, i}-\mathrm{E}\left[\xi_{\mathrm{CNint}, i}-\xi_{\mathrm{CNres}, i}\right]\right\|_{2}^{-1}$ energy balance degree $\eta$ will be obtained.

\section{Simulation Results and Analysis}

In order to verify the correctness of the algorithm and calculate the energy consumption of network nodes, the algorithm is simulated and analyzed in this section.

Figure 1 shows the traditional cooperative MISO scheme, the propsed scheme and the ideal feedback cooperative MISO transmission scheme in sending the same amount of data, energy consumption balance is affected by the 
number of clusters in the graph CNs. Figure 1 shows that the increase of the traditional collaborative MISO scheme with energy balance in the cluster CNs number decreases, but the decrease was smaller, that is to say, the number of CNs cluster has little effect on the energy consumption of the nodes in the network equilibrium degree, and this section proposed scheme of balanced energy consumption with the increase in the cluster number $\mathrm{CNs}$ the increase of the energy consumption of nodes in the network is balanced, in addition, the scheme of balanced energy consumption was about the traditional collaborative MISO scheme of balanced energy consumption of 5 times, for the balance of energy consumption is nearly complete with cooperative MISO transmission state information feedback (perfect CSI for short) energy balance is a suboptimal scheme. In summary, the proposed scheme can greatly improve the node energy balance, the energy consumption of nodes in the network is relatively balanced, so as to prolong the network lifetime, cluster and CNs number on node energy balance degree has a great influence.

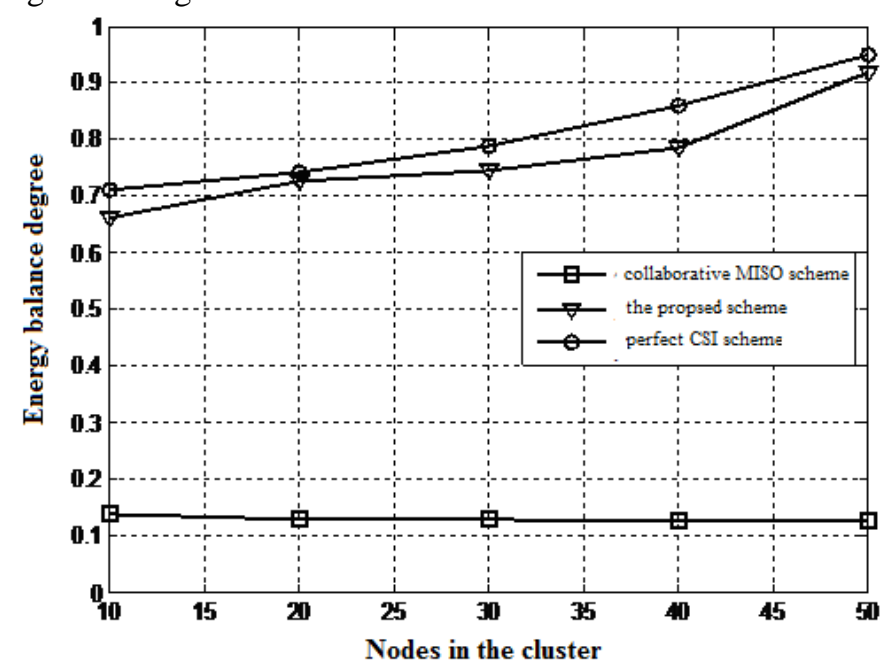

Fig. 1 Comparison of the performance for the network energy consumption Theorems and Proofs

\section{Conclusion}

This paper presents a method for calculating equilibrium degree of the node energy consumption and its balance scheme. Theoretical analysis and simulation results show that in wireless sensor network, comparing with the traditional cooperative MISO scheme and the ideal feedback cooperative scheme, energy consumption balance algorithm can reduce energy consumption and improve 
energy balance degree. Energy consumption of nodes in the network is relatively balanced, and the network life time can be also extended.

\section{Acknowledgments}

This work was financially supported by the Shaanxi Nature Science Foundation (2015JM6325 and 2014JM26101).

\section{References}

1. Xue Y, Gershman A B. Alamouti-type wireless communication systems with one-bit feedback [J]. IEEE Sensor Array and Multichannel Signal Processing Workshop, 2004, 7:143-147.

2. Goldsmith A, Jafar S A, Jindal N, et al. Capacity limits of MIMO channels[J]. IEEE Journal on Selected Areas in Communications, 2003, 21(5):684-702.

3. Love D J, Heath Jr R W, Santipach W, et al. What is the value of limited feedback for MIMO channels[J]? IEEE Communications Magazine, 2004, 42: 54-59.

4. Q. Bai and J. Nossek, Energy efficiency maximization for 5G multi-antenna receivers [J]. Transactions on Emerging Telecommunications Technologies, 2015, 26(1):3-14.

5. B. Murmann, Energy limits in A/D converters[C], in Faible Tension Faible Consommation (FTFC), 2013 IEEE, June 2013, pp. 1-4. 